IP Periodica Polytechnica

Mechanical Engineering

59(3), pp. 137-142, 2015

DOI: 10.3311/PPme.7998

Creative Commons Attribution (i)

RESEARCH ARTICLE

\section{A Novel Floor Sensor Network Technology Based on Dielectric Electro-Active Polymers}

\author{
Ákos Pintér ${ }^{*}$
}

Received 22 February 2015; accepted after revision 14 May 2015

\begin{abstract}
This study describes a novel floor sensor network being developed to provide a competitive and universal pressure mapping technology for a wide range of medical, research and security applications. The presented sensor network consists of 16 sensor elements distributed in a $4 \times 4$ sensor matrix. The sensor elements are made of a special dielectric electro-active polymer film being capable of capacitive sensing of the surface pressure. Each sensor unit is $200 \times 160 \mathrm{~mm}$ resulting in an overall sensor matrix size of $800 \times 740 \mathrm{~mm}$. A high precision and high speed sensor interface was realized to provide a differential capacitance measurement method fulfilling the specific measuring requirements of the sensor matrix. Based on the measurement results, the developed sensor network is capable of measuring the distributed weight on their surface with an accuracy of $5 \mathrm{~kg}$, however, this initial measuring accuracy can be significantly increased by improving the manufacturing technology of the sensor elements.
\end{abstract}

\section{Keywords}

surveillance monitoring, pressure sensor, thin film sensors, capacitive sensor, dielectric films, capacitance measurement

\section{Introduction}

Pressure mapping is an innovative technology providing a solution for a wide range of applications that has been available for many years but is still not widespread owing to the high prices of the used sensor materials.

A pressure mapping system is an array of sensors contained in a flexible mat that measure interface pressure, which is defined as the pressure that occurs at the interface between the body and the support surface. Depending on the application, the surface pressure being distributed on the sensor matrix can be monitored or processed by special algorithms to make conclusions and decisions in order to trigger a control, warning or alarm signal.

Pressure mapping systems are used by product designers and test engineers throughout the research, design, testing, and manufacturing cycle to verify and enhance product performance. The informative data allows companies to save money in design, design verification, reengineering and machine setup costs. Some examples of the possible application fields including general purpose pressure mapping, seating and mattress pressure mapping, tire tread pressure measurement, wiper force measurement and ergonomic pressure measurement are shown on Fig. 1.

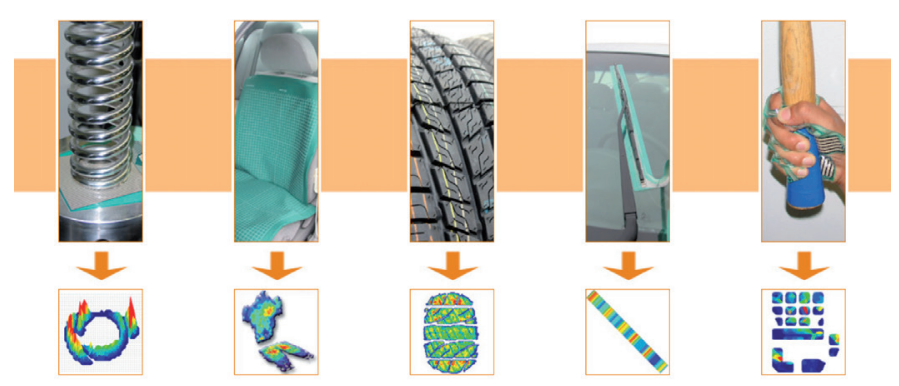

Fig. 1 Applications of pressure mapping systems [2]

Pressure mapping systems are also used for patient care and medical researchers. Some medical studies and applications including in-shoe pressure analysis, barefoot pressure analysis, seating and positioning pressure analysis, joint pressure analysis and animal gait pressure analysis are shown on Fig. 2.

\footnotetext{
${ }^{1}$ Department of Mechatronics, Optics and Information Engineering, Faculty of Mechanical Engineering,

Budapest University of Technology and Economics,

1111 Budapest, Hungary
}

*Corresponding author, e-mail: akospinter@gmail.com, akos.pinter@de.bosch.com 


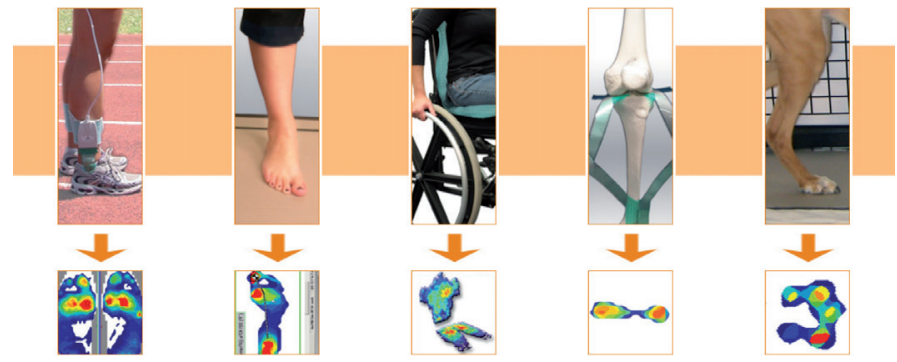

Fig. 2 Applications of pressure mapping systems [2]

\section{Research objectives}

The main objective of the presented research project was to develop a competitive and universal floor sensor network which provides a platform technology for a wide range of medical, research and security applications. The major milestones of the project were the determination of the proper sensor material, the development of a high precision and high speed evaluation electronics, the construction of a prototypic floor sensor network and the development of basic software functions which controls the evaluation electronics, processes the output signals of the electronics and visualizes the distributed weight on each sensor elements with the aid of a graphic-interface.

\section{Sensor matrix}

\subsection{Choosing the sensor material}

Pressure mapping systems are highly dependent on the material properties of the pressure transducer. The most important properties of a sensor element are the scalability (which allows to fit the geometrical resolution according to the application specific requirements), the sensor linearity (which allows a weight measurement independent from the pressure distribution on the elements) and the sensor respectively noise sensitivity (which allows the desired measuring precision of $1 \mathrm{~kg}$ ). The specific cost of the sensor material, the manufacturing costs of the sensor matrix respectively the necessary evaluation electronics and the patent situation are also important in order to provide a competitive technology.

In order to find the proper sensor material a literature research regarding the available pressure sensitive materials was done. A detailed analysis and evaluation based on the above mentioned requirements was performed on a pre-selection of the considerable sensor materials (resistive sensor material [2], piezoelectric sensor material [3], real-charge electrets material [4], electro-active polymer material [5]). The analysis of the sensor materials is not treated in this paper. Related work can be found in [1]. Based on the results of the analysis a particular DEAP (dielectric electro active polymer) material working on a capacitive principle was chosen.

DEAP is an elastomeric polymer sheet or silicon film coated on both sides with conductive material which act as electrodes. A sheet of the material is equivalent to a parallel plate capacitor and its capacitance is dependent on the area of the electrodes and the distance between them. These geometries are altered by applying mechanical stress on the sheet resulting in a changing capacitance, thus such films can be used as a capacitive displacement or pressure sensor.

Danfoss PolyPower A/S develops and manufactures a special optimized DEAP material [5]. The PolyPower DEAP film consists of a thin silicone dielectric material $(\sim 40 \mu \mathrm{m})$ with a special corrugated surface $(\sim 7 \mu \mathrm{m})$ and very thin layer of compliant metal electrodes $(\sim 100 \mathrm{~nm})$ on top of it. During the rollto-roll manufacturing, two sheets of on one side corrugated and metallised silicon films are laminated together resulting in the patented design (Fig. 3), which ensures significantly higher performance for both sensor and actuator applications than other alternative design of DEAP materials.
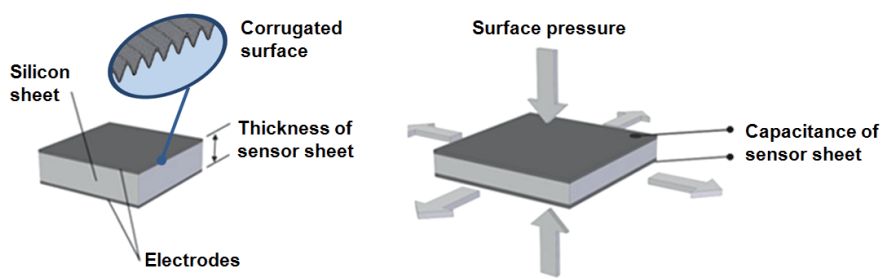

Fig. 3 Polypower DEAP

Surface pressure can be measured when the compliant PolyPower sensor material is configured for thickness mode. Due to the special corrugated surface the measured capacitance change is linearly proportional with the applied pressure [1]. In addition, the used scalable roll-to-roll manufacturing technology allows for volume production.

\subsection{Preparation of the sensor matrix}

The Danfoss PolyPower DEAP film is manufactured with a scalable roll-to-roll technology, thus the raw material was available in $5 \mathrm{~m} \times 0,2 \mathrm{~m}$ rolls. All the 16 sensor units having a size of 200x160 mm each were prepared on a single sheet roll. The prepared sheet roll was cut into smaller pieces consisting of 4 sensor units each and these pieces were mounted together forming a $4 \times 4$ sensor matrix being shown on Fig. 4 .

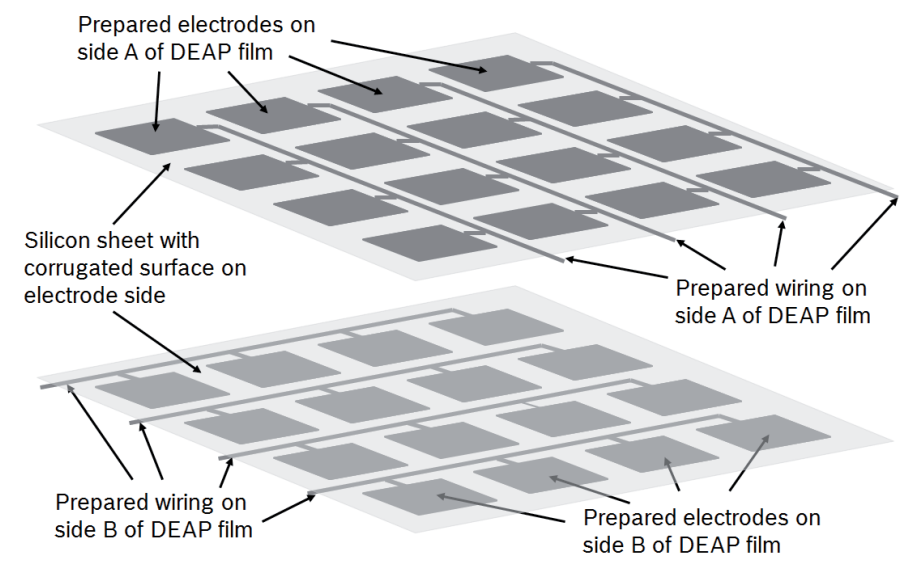

Fig. 4 Layers of prepared DEAP film 
The sensor electrodes and wiring were prepared by removing the unwanted electrodes with the aid of an oxidizing agent on both side of the DEAP film (sodium hypochlorite). The metal electrodes are very fragile and can easily be damaged. In addition, the large electrode surface makes the sensor network susceptible for electromagnetic interferences, which would limit the possible measuring accuracy. In order to resolve these problems, some additional layers were applied for the prototypic sensor build-up as shown on Fig. 5.

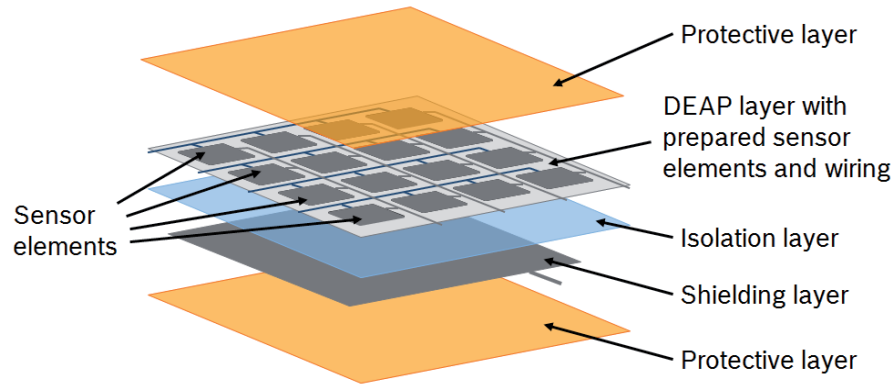

Fig. 5 Sensor matrix built-up

The prepared DEAP film was laminated with a self-adhesive polypropylene film (isolation layer) and an aluminium foil (shielding layer) which has to be grounded. Additionally, the laminated sensor matrix have been covered with a thin soft foam material on the upper side and with a further polypropylene film on the bottom side which provides for a better mechanical protection. The laminated sensor network excluding the protective layers is shown on Fig. 6.

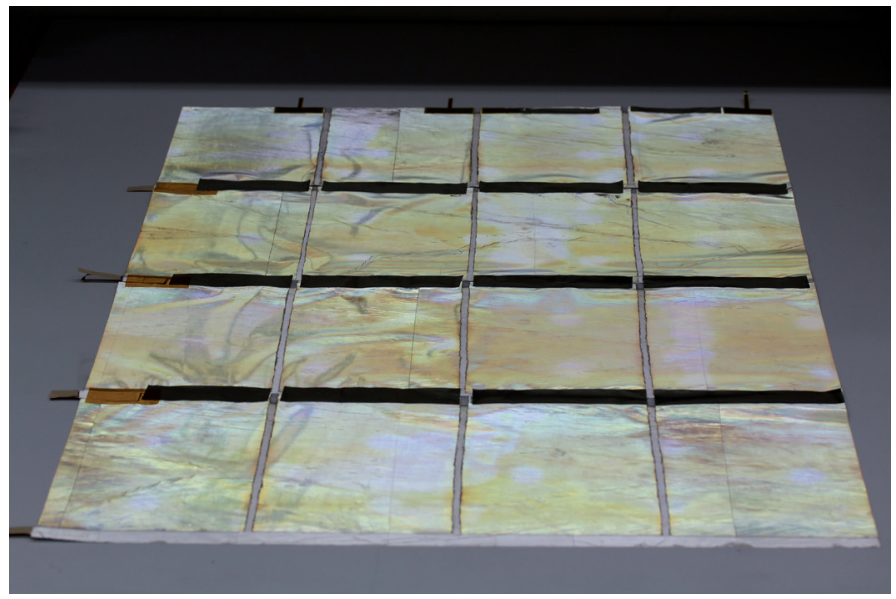

Fig. 6 Sensor matrix excluding protective layers

For the electrical contacting of the row and column electrodes a self-adhesive conductive tape was used.

\section{Sensor interface}

\subsection{Scanning method}

When applying a sensor structure such as the one shown in Fig. 4, there are many crosstalk currents passing through sensor units other than the actual measured sensor element. These currents can interfere with the measurement as shown in Fig. 7.

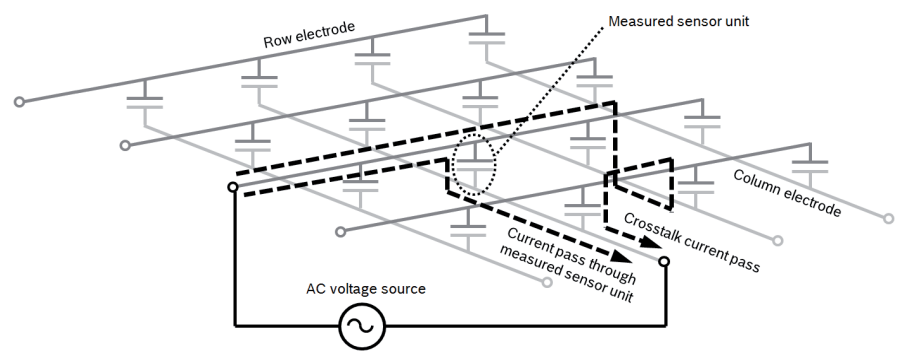

Fig. 7 Crosstalk currents in case of not properly scanned sensor matrix

There are several methods of preventing these surrounding currents. The sensor elements could be measured completely independently [6]. However, by this method, field effect transistor (FET) switching is performed at every sensor element, which not allows achieving a thin and flexible measuring component. Snyder et al. [7] proposed the method of preventing these crosstalk currents by inserting diodes at all of the measuring points. However, this method cannot be used for capacitive sensor elements where the excitation signal is an AC signal. In addition, Purbrick proposed the current mirror method [8]. This method eliminates crosstalk current passes by setting the drive lines that are not a consideration for the measured sensor unit at a potential equal to the output voltage. Similar to this method, Hillis proposed the zero potential method [9]. By changing the output circuit of the voltage mirror method, the voltage of the scanning electrodes is set to a zero potential resulting in a simplified circuitry.

The scanning method used in the proposed floor sensor network is designed by taking the above points into consideration. In Fig. 8, the conceptual diagram of the scanning circuit is shown.

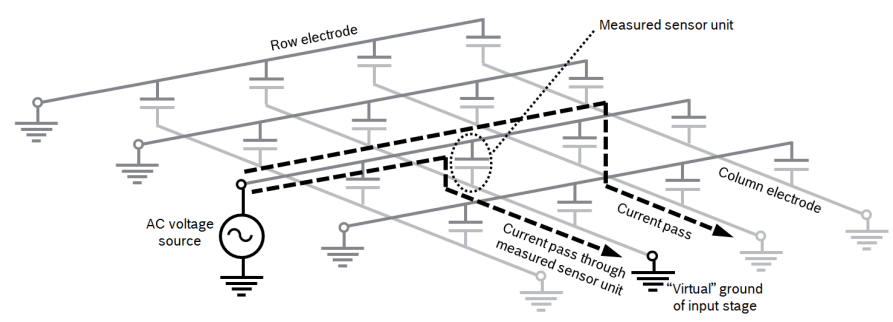

Fig. 8 Crosstalk currents in case of zero potential scanning

Excitation voltage is applied to the row to be selected while the other rows are brought to zero voltage. As for column direction, the input stage of capacitance measuring circuit (virtual ground) was connected to the column to be selected and other columns are brought to zero voltage as well. Applying the zero potential scanning method, the measurement error owing to crosstalk currents electrodes can be excluded.

\subsection{Measurement method and circuit}

A main milestone of the floor sensor network project was the development of a high precision and high speed evaluation electronics (being capable of handling 1000 sensor units and of detecting capacitance changes in the order of $100 \mathrm{ppm}$ of 


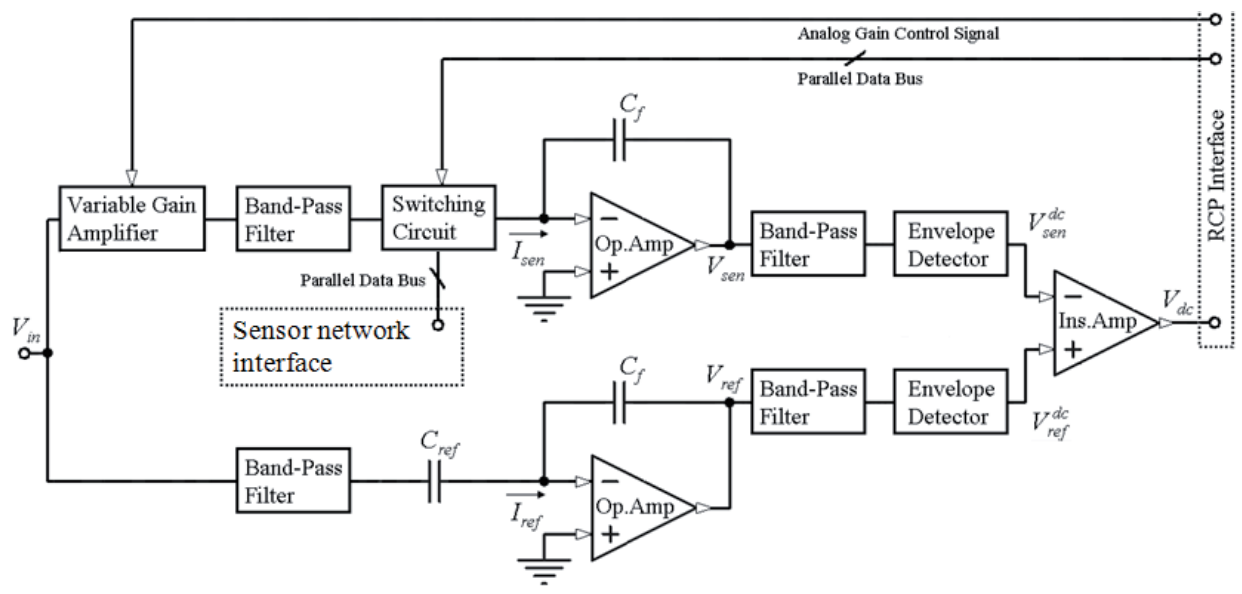

Fig. 9 Capacitance measurement circuit

the nominal capacitance value with a sampling frequency of $2 \mathrm{kHz}$ ). On the basis of literature research, a state-of-art and a novel measurement circuit were developed [10-11]. As conclusion of this study a conventional bridge measurement [12-13] method has been chosen for the first technology demonstrator. The block diagram demonstrating the operation principle is shown in Fig. 9.

The used bridge circuit consists of two current-to-voltage converters, forming the two branches of the measuring-bridge, and of an instrumentation amplifier observing and gaining the amplitude difference of the signals. The operational amplifier as well as the instrumentation amplifier was chosen with an upper corner frequency slightly above the frequency of the carrier signal (acting as low-pass filters) to achieve good high frequency noise suppression.

The high-pass filters were implemented to reduce and eliminate the effect of the low-frequency noises, even the effect of noises having a frequency content in the same order of magnitudes as the carrier frequency itself. For this purpose a fourthorder Chebyshev high-pass filter was used. The Chebyshev high-pass filter provides a rather high gain rolloff below the cutoff frequency with the drawback of having magnitude ripples in the passband. Since in case of an amplitude modulated measuring system, the operating frequency is a single frequency, this drawback is rather irrelevant.

Due to practical experiences, a half wave rectifier is having less ringing than the full wave rectifiers, making this topology more suitable for our purposes. Two major requirements are considered at the dynamic design of the envelop detector: on one hand the settling time of the controlled circuit cannot be longer as ten times the period time of the carrier frequency (100 $\mathrm{kHz}$ ), on the other hand the carrier signal have to be filtered out at least with $80 \mathrm{~dB}$. In order to satisfy the above conditions, a sixth-order Bessel low-pass filter was implemented. The Bessel low-pass filters have a linear phase response over a wide frequency domain, resulting in a constant group delay in that range. Due to this reason, Bessel low-pass filters provide optimum square-wave transmission behaviour, which is important in order to have a fast settling time of the resultant system.

The good noise suppression of the circuit allows applying a higher gain on the instrumentation amplifier which provides the capability of measuring very small capacitance changes. However, the higher is the measuring gain, the smaller is the measuring range. To be able to apply the highest possible gain, the bridge circuit is balanced for each unloaded sensor elements by a sensor-individual adjustment of the excitation signal. The sensor-individual balancing is performed with the aid of a variable gain amplifier.

The switching circuit performing the zero potential scanning consists of 8 analogue three state switches (signal, open and ground) being connected to the control unit over an 8-bit parallel data bus.

\section{Experimental setup and results}

The complete experimental setup is shown on Fig. 10.

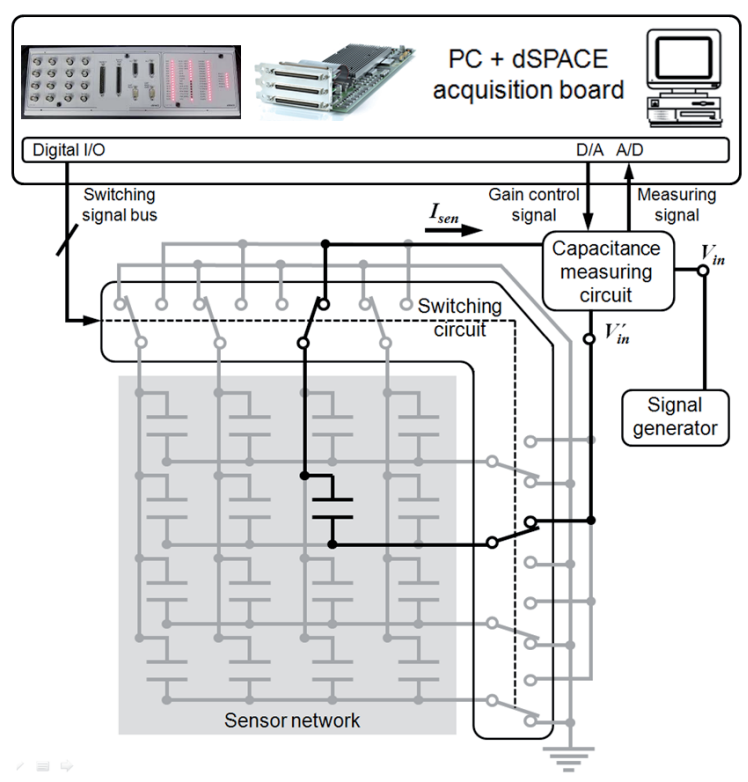

Fig. 10 Experimental setup 
The basic functionality as control of zero potential scanning, sensor-individual adjustment of excitation signal and the visualization of the loading state of each sensor elements were modelled in MATLAB Simulink and implemented in a dDPACE rapid control prototyping system.

The sensor elements are scanned sequentially using the zero potential scanning method. The sensor-specific amplitude of excitation signal is set for each sensor units by an analogue gain control signal. The gain control voltages are determined by a onetime balancing of the unloaded sensor units. The measuring signal is captured and converted to physical signal (surface load) after the settling time is elapsed. The registered loading values are visualised with the graphical interface of the rapid control prototyping software as shown on Fig. 11.
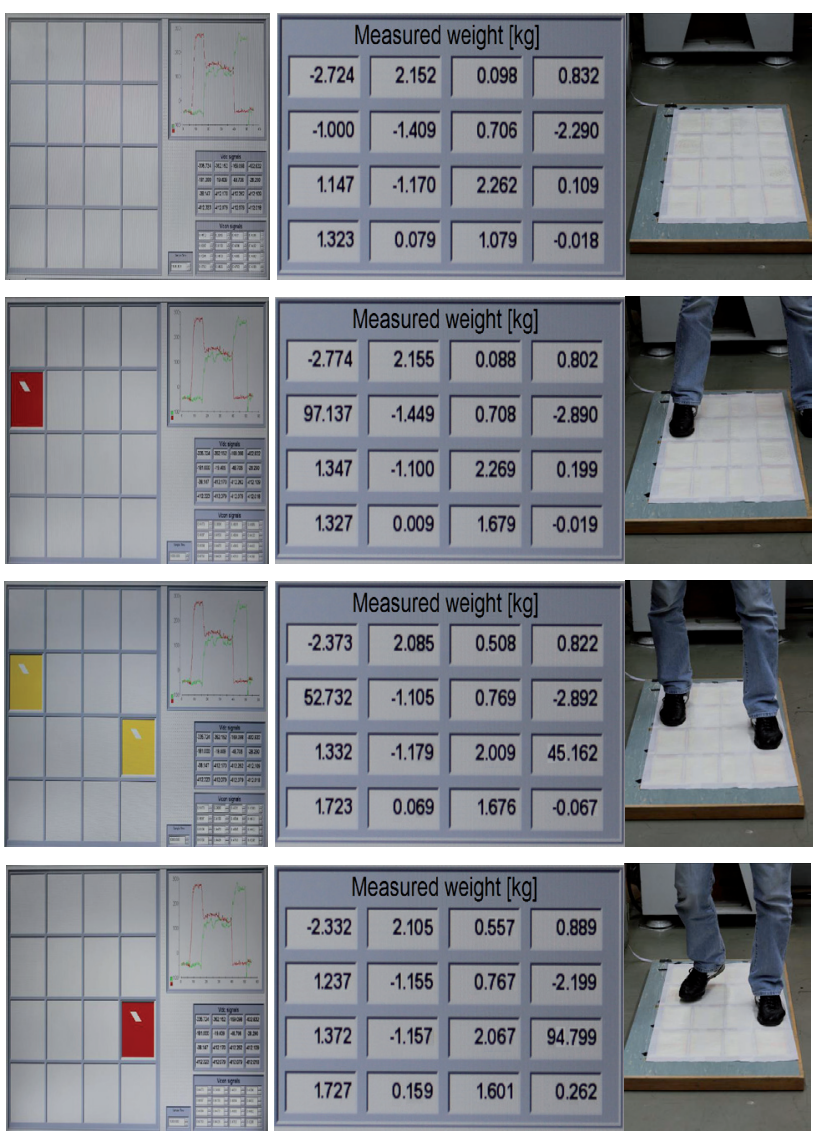

Fig. 11 Floor sensor network system in operation

The measured values are visualized on both optical (the different loading states were represented with different colours on a virtual sensor network) and numeric way (the actual measured values were shown on a numeric matrix). Above measurement was done a few days after the balancing of the circuit with unloaded sensor elements. The loading values of the unloaded sensors are representing the medium-term drift of the sensor elements (in the range of few kilograms). This medium-term drift can be eliminated by a continuous balancing of the measurement circuit. Additionally, the loading state of two elements was registered in time to study the dynamical deviations of the measurement as shown in Fig. 12.

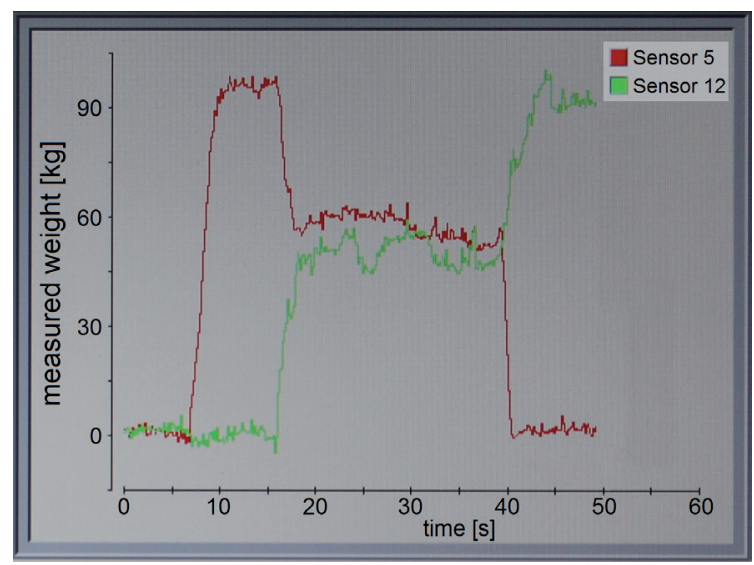

Fig. 12 Weight-time diagrams of the loaded sensor units

The registered weight-time diagrams of the two loaded sensors are showing some unexpected jumps of the signal (in the range of $5 \mathrm{~kg}$ ) in the loaded states. These disturbances are arising from the manufacturing errors of the sensor elements (e.g. remaining air voids during lamination of additional layers) owing to the lack of professional manufacturing technology.

\section{Conclusion}

In this study, a thin, flexible, sheet-like floor sensor network and corresponding measuring/evaluating electronics were developed. Instead of the conventional pressure sensitive materials such resistive, piezoelectric or real-charge electrets films, a much cheaper material was used. The current market prices of the studied materials are shown in Table 1.

Table 1 Specific costs of studied sensor materials

\begin{tabular}{cc}
\hline Sensor material and manufacturer & Specific costs $[\mathbf{€} / \mathbf{m} 2]$ \\
\hline $\begin{array}{c}\text { Pressure sensitive conductive rubber sheet } \\
\text { by Tekscan Inc. }\end{array}$ & $\sim 880$ \\
\hline $\begin{array}{c}\text { Metalized piezoelectric PVDF film } \\
\text { by Measurement Specialities Inc. }\end{array}$ & $\sim 2150$ \\
Real-charge electrets film \\
by Emfit Ltd.
\end{tabular}

Furthermore, the applied roll-to-roll manufacturing technology and the lower raw material costs of DEAP (in comparison to the other sensor materials) allow a significant decrease of specific costs in case of larger production volumes providing a competitive technology for industrial applications (e.g. surveillance or patient monitoring). The first technology demonstrator was able to detect the movement of a person and to register the weight distribution on its surface with an accuracy of $5 \mathrm{~kg}$. 
After improving the manufacturing process of the sensor network (which is supposed to increase the measuring accuracy) and increasing the number of sensor units, the implementation of higher level functions (e.g. trajectory recognition, personal identification, weight analysis) can be realised. An improved floor sensor network could provide a technology platform for developing advanced applications (e. g. orthopaedic step analysis, fall or intrusion detection).

\section{Acknowledgement}

The study presented in this article is supported by the Central Research Department of Robert Bosch GbmH.

\section{References}

[1] Pintér, Á. "Nyomásérzékeny szenzoros anyagok karakterizálása." (Characterization of pressure sensitive materials.) In: OGÉT XXII. Nemzetközi Gépészeti Találkozó. Nagyszeben. pp. 289-296. 2014. (in Hungarian)

[2] Tekscan, Inc. Pressure and Force Measurement Applications. [Online] Available from: https://www.tekscan.com/applications [Accessed: 12th May 2015]

[3] Measurement Specialities Inc. Piezo Film Sensors, Technical Manual, PM 1005663-1 REV 1302 APR 99

[4] Paajanen, M., Lekkala, J., Valimaki, H. "Electromechanical modeling and properties of the electret film EMFI." IEEE Transactions on Dielectrics and Electrical Insulation. 8 (4). pp. 629-636. 2001.

DOI: $10.1109 / 94.946715$

[5] Jones, R.W., Wang, P., Lassen, B., Sarban, R. "Dielectric elastomers and compliant metal electrode technology." In: 15th IEEE Mediterranean Electrotech. Conference (MELECON). 26-28 April 2010. pp. 368-373. 2010. DOI: 10.1109/MELCON.2010.5476257

[6] Shimojo, M., Ishikawa, M. "An imaging tactile sensor with video output and tactile image processing." Transactions of the Society of Instrument and Control Engineers. 24 (7). pp. 662-669. 1988.

DOI: $10.9746 /$ sicetr1965.24.662

[7] Snyder, W. E., St. Clair, J. "Conductive elastomers as sensor for industrial parts handling equipment." IEEE Transactions on Instrumentation and Measurement. 27 (1). pp. 94-99. 1978.

DOI: $10.1109 /$ TIM.1978.4314628
[8] Purbrick, J. A. "A force transducer employing conductive silicone rubber." Proc. 1st Int. Conf. Robot Vision and Sensory Controls. pp. 73-80. 1981.

[9] Hillis, W. D. "A high-resolution imaging touch sensor." International Journal of Robotics Research. 1 (2). pp. 33-44. 1982. DOI: $10.1177 / 027836498200100202$

[10] Pinter, A., Denes, I. "Interface Circuit for Measuring Small Capacitance Changes in Sensor Networks." Science, Measurement \& Technology. 2015. DOI: 10.1049/iet-smt.2014.0221

[11] Pinter, A., Denes, I. "Stability Issues on an Implemented All-Pass Filter Circuitry." Proceedings of World Academy of Science, Engineering and Technology. 6 (3). pp. 614-620. 2011.

[12] Preethichandra, D. M. G., Shida, K. "A Simple Interface Circuit to Measure Very Small Capacitance Changes in Capacitive Sensors." IEEE Transactions on Instrumentation and Measurement. 50 (6). pp. 15831586. 2001. DOI: $10.1109 / 19.982949$

[13] Haider, M. R., Mahfouz, M. R., Islam, S. K., Eliza, S. A., Qu, W., Pritchard, E. "A Low-power Capacitance Measurement Circuit With High Resolution and High Degree of Linearity." In: 51st Midwest Symposium on Circuits and Systems. pp. 261-264. 2008. DOI: 10.1109/MWSCAS.2008.4616786

[14] Iskandarani, Y., Karimi, H. R. "Pressure sensor development based on Dielectric Electro Active Polymers." In: 7th IEEE Conference on Industrial Electronics and Applications (ICIEA). pp. 530-535. 2012. DOI: 10.1109/ICIEA.2012.6360785

[15] Iskandarani, Y., Karimi, H. R. "Sensing Capabilities Based on Dielectric Electro Active Polymers-Feasibility and Potential State-of-the-Art Application." IEEE Sensors Journal. 12 (8). pp. 2616-2624. 2012. DOI: 10.1109/JSEN.2012.2197198

[16] Shimojo, M., Namiki, A., Ishikawa, M., Makino, R., Mabuchi, K. "A Tactile Sensor Sheet Using Pressure Conductive Rubber With ElectricalWires Stitched Method." IEEE Sensors Journal. 4 (5). pp. 589-596. 2004. DOI: 10.1109/JSEN.2004.833152

[17] Kiil, H. E., Benslimane, M. "Scalable industrial manufacturing of DEAP." Proc. SPIE EAPAD. 7287. pp. 72870R-1-72870R-10. 2009. DOI: $10.1117 / 12.815741$ 